\title{
Bewusstsein - Reichweite und Grenzen naturwissenschaftlicher Erklärung
}

\author{
Alfred Gierer \\ Max-Planck-Institut für Entwicklungsbiologie, Spemannstrasse 35, D-72076 Tübingen
}

Beitrag zur Arbeitsgruppe "Humanprojekt - Die Stellung des Menschen in der Natur" der Berlin-Brandenburgischen Akademie der Wissenschaften. In: Funktionen des Bewusstseins, Humanprojekt/Interdisziplinäre Anthropologie 2 (D. Ganten, V. Gerhardt, J. Nida-Rümelin, Hg.), S. 267-282, de Gruyter, Berlin 2008.

\section{Zusammenfassung}

Nach einem kurzen Resümee über entscheidungstheoretisch begründete Grenzen einer rein naturwissenschaftlichen Theorie menschlichen Bewusstseins werden Implikationen für unser Menschenbild erörtert. Was die Willensfreiheit angeht, so sprechen starke erkenntnistheoretische Gründe dagegen, dass der Wille Anderer durch Analyse von Außen vollständig erschließbar sein könnte; was aber für niemanden determiniert ist, ist nicht determiniert. Für die Evolution mentaler Fähigkeiten, die den biologisch modernen Menschentyp charakterisieren, könnten auch einzelne, eher seltene Neukombinationen von Abschnitten im Genom eine wesentliche Rolle gespielt haben. Die entwickelten Fähigkeiten und Eigenschaften führten dann weit über den Anlass ihrer Entstehung hinaus. Hinsichtlich der menschlichen Erkenntnisfähigkeit ist die grundsätzliche philosophische Frage, ob und wieweit es eine Ordnung der Welt lediglich als Konstrukte unserer Gehirne, oder aber auch vor und ohne uns gibt, rein neurobiologisch sicher nicht zu entscheiden. Dies betrifft zum Beispiel das Grundverständnis der Mathematik, aber auch das von Religion. Naturwissenschaften insgesamt und ihre wissenschaftlich begründeten Grenzen sind mit verschiedenen, natürlich nicht mit allen philosophischen, kulturellen und religiösen Deutungen vereinbar
Gehirn-Geist-Beziehung: Gründe für Grenzen der Dekodierbarkeit

Bewusstsein ist kein Begriff physikalisch begründeter Naturwissenschaften, und doch ist menschliches Bewusstsein Voraussetzung dafür, dass es Naturwissenschaften überhaupt gibt. Was ist Bewusstsein? Eine Klärung ist aus einer Reihe tiefliegender Gründe schwierig. Zum einen sind Grenzen etwa im Vergleich von Mensch und Tier schwer zu ziehen. Zum anderen einigt man sich, was menschliches Bewusstsein angeht, ziemlich leicht auf einige notwendige Kriterien, zum Beispiel den Selbstbezug; eine hinreichende Liste von Kriterien dafür, wer oder was Bewusstsein hat, ist aber kaum realisierbar, ist vielleicht prinzipiell unmöglich. Dazu folgendes Gedankenexperiment: Nehmen wir an, wir hätten eine Liste von formalen Merkmalen, die wir für vollständig erklären, und würden sie in einer computer-gesteuerten Puppe implementieren. Würden wir diese dann einvernehmlich und mit allen - auch den juristischen und moralischen - Konsequenzen als im menschlichen Sinne bewusst anerkennen? Kaum.

Zweifellos ist Bewusstsein eine Funktion von Gehirnprozessen, und die unterliegen den Grundgesetzen der Physik. Was formalierbar ist, ist mechanisierbar; wir dürfen daher vermuten, dass jede, auch jede höhere, Gehirnfähigkeit, die sich vollständig formal beschreiben lässt, schliesslich einer naturwissenschaftlichen Erklärung zugänglich sein sollte. Es ist aber doch zu fragen, ob es nicht prinzipielle Grenzen der Formalisierung von 
Bewusstseinsvorgängen und Zuständen gibt, wie dies ja schon durch die Definitionsprobleme nahegelegt wird.

Zwei Grundeinstellungen verdienen nach meiner Ansicht bei der Suche nach Antworten besonderes Vertrauen: Erstens konsequenter Physikalismus: Die Physik gilt ohne Einschränkung für alle Ereignisse in Raum und Zeit, Gehirnprozesse eingeschlossen; zweitens aber auch epistemologisch begründete, entscheidungstheoretische Skepsis: Es gibt prinzipielle Grenzen der Berechenbarkeit und Entscheidbarkeit, wie sie Heisenbergs Unbestimmtheit für die Physik und Gödels Theoreme für die Mathematik eindrucksvoll aufgezeigt haben. Nicht, dass daraus direkt etwas für die Gehirn-Geist-Beziehung folgen würde; wesentlich ist aber, dass überhaupt mit grundsätzlichen, wissenschaftlich begründbaren Erkenntnisgrenzen zu rechnen ist, zumal wenn, wie schon in der Physik und Mathematik, Selbstbezug involviert ist. Insbesondere zeigt die mathematische Entscheidungstheorie, dass kein einigermassen leistungsfähiges logisches System mit den je eigenen Mitteln gegen Widersprüche abgesichert werden kann. Ich meine, dass sich in Analogie hierzu besonders selbstbezogene Aspekte von Bewusstsein einer vollständigen algorithmischen Theorie entziehen könnten.

Nun gelten zwar die Theoreme mathematischer Unentscheidbarkeit für unendliche Gegebenheiten, während unser Gehirn in seinen Möglichkeiten ein endliches System ist, sodass man im Prinzip über die Gültigkeit jeder allgemeinen Aussage entscheiden könnte, indem man alle Möglichkeiten hintereinander durchspielt und überprüft. In Wirklichkeit folgt aus mathematischer Endlichkeit aber noch lange nicht Ableitbarkeit mit innerweltlichen Mitteln. Die sind nämlich aus fundamentalen physikalischen Gründen naturgesetzlich begrenzt - selbst bei grosszügiger Abschätzung auf unter $10^{120}$ Rechenschritte. Das sind nun keineswegs irgendwelche Zahlen; sie hängen stringent $\mathrm{ab}$ von den Dimensionen des Universums und eng mit den Grundkonstanten der Physik zusammen, und das spricht dafür, solche Begrenzungen erkenntnistheoretisch ernstzunehmen: Die Endlichkeit der Welt begrenzt auch die Entscheidbarkeit von Problemen; was nur für einen superkosmi- schen Computer determiniert wäre, ist nicht determiniert. Nun reicht aber selbst eine so hohe Anzahl realisierbarer Rechenoperationen wie $10^{120}$ nicht unbedingt aus, wenn es um die Analyse solch weiter Felder von Möglichkeiten geht, wie sie erforderlich ist, wenn man aus der Datenflut der Gehirnzustände zum Beispiel selbstbezogene Verhaltensdispositionen für eine offene Zukunft verlässlich ableiten will; man kann sie nicht alle nacheinander testen, um herauszufinden, welche Disposition einem physikalischen Gehirnzustand nun entspricht.

Natürlich kann man trotzdem durch systematische Forschung - durch bewusstseinsnahe Neurobiologie, durch Psychophysik, auch durch theoretische Modelle - sehr Vieles und sehr Interessantes über die Gehirn-Geist-Beziehung herausbekommen; aber es gibt eben keine Garantie, keinen Algorithmus für Antworten auf jede vernünftige Frage, auch nicht auf jede Frage nach psychischen Zuständen und Dispositionen. Vielmehr ist es eine begründete Vermutung, dass es prinzipielle Grenzen der Dekodierbarkeit der Beziehung zwischen neurophysiologischen und psychischen Zuständen gibt, zumal wenn Selbstbezug im Spiel ist.

\section{Funktionen des Bewusstseins und deren Evolution}

Angesichts dieser Grenzen sollte man nun nicht die bedeutenden wissenschaftlichen, zumal neurobiologischen Erkenntnisse zum besseren Verständnis des Bewusstseinsproblems übersehen. Menschliches Bewusstsein ist besonders durch vier Fähigkeiten charakterisiert: Die Fähigkeit der Bindung sehr verschiedener Aspekte der Wahrnehmung und des Wissens zu einer möglichst guten Interpretation einer Gesamtsituation; die Fähigkeit der Abstraktion, die auch Symbolik und Meta-Ebenen des Denkens ermöglicht, zumal in Zusammenhang mit der menschlichen Sprache; die Fähigkeit der kognitionsgestützte Empathie, die es erlaubt, sich in die Gefühle, das Wissen und die Vorhaben Anderer hineinzuversetzen; und die Fähigkeit der Zeitintegration, die Verfügung über Erinnerungen auch in eine ferne Vergangenheit ebenso wie die Vorausschau in Handlungsmöglichkeiten in einem weiten Zukunftshorizont. All die Fähigkeiten, die Ge- 
genstand hochinteressanter neurobiologischer Forschung sind, bündeln sich in der Basisfähigkeit, die nach meiner Auffassung den biologisch modernen Menschentyp charakterisiert: Das umfassende strategische Denken.

Dieser moderne Menschentyp Homo sapiens sapiens ging vermutlich vor etwa 200000 Jahren aus einer kleinen Menschengruppe in Afrika hervor; er hat danach alle anderen Menschentypen verdrängt und schliesslich, seit über 30000 Jahren, in die Eigendynamik der Kulturgeschichte geführt. Seine umfassende Kulturfähigkeit ist ein Produkt biologischer Evolution, die einzelne Kultur ist es nicht. Nach einer Phase der Ko-Evolution genetischer und kultureller Merkmale konnte die kulturelle Differenzierung und Entwicklung bis hin zu den Hochkulturen dann wohl ohne wesentliche Genänderungen erfolgen, die mentale Fähigkeiten betrafen.

Wir wissen nicht, welche Mutationen es waren, die in der Evolution bei der biologischen Begründung der Kulturfähigkeit des modernen Menschentyps eine wesentliche Rolle gespielt haben. Vorherrschende Meinung ist, dass dies in einer Vielzahl von für sich betrachtet recht unspezifischen Schritten geschah. Die Systemtheorie zeigt, dass in der Tat auf diese Weise Umschlag von Quantität in Qualität möglich ist, eine Art ,Selbstorganisation“, die zur Ausbildung neuer Strukturen und Funktionen führen kann. Es ist der intellektuelle Charme der Selbstorganisation, vielleicht auch der vermeintlichen, als besonders aufgeklärt geltenden Distanz zur Schöpfungstheologie, die der extremen Kontinuitätstheorie psychologische Vorteile in der wissenschaftlichen Community verschafft. Aber Vorsicht: Es könnten auch wenige Genänderungen, es könnte sogar eine einzelne, singuläre genetische Mutation eine neue Richtung der Evolution begründet haben; eine Richtung, die z.B. an der Ausbildung des neuralen Netzwerkes ansetzte, indem in weiten Bereichen der Grosshirnrinde solche Verschaltungen der Neurone ausgebildet wurden, die neue Meta-Ebenen der Informationsverarbeitung ermöglichten oder sehr stark erleichterten. Auf derartigen erweiterten Fähigkeiten wiederum beruht letztlich das ganze Bündel von Faktoren, das für menschliches strategisches Denken erforderlich ist.
Dazu gehört die Anwendung analytischer Prozesse auf analytische Prozesse selbst. Dazu gehören nicht zuletzt auch multiple Selbstrepräsentationen im Gehirn in Form abstrakter „Selbstbilder“, in denen mögliche Zustände der eigenen Person in verschiedenen Szenarien der Zukunft repräsentiert sind und die darstellen, wie wir sind, wie wir werden und nicht werden wollen. Als Voraussetzung menschlicher, kognitionsgestützter Empathie gehören dazu auch die Repräsentationen Anderer, die deren Befindlichkeiten, Erwartungen und Befürchtungen für die $\mathrm{Zu}$ kunft einschliessen. Empathie ist ganz wesentlich für die eindrucksvolle Kooperationsfähigkeit unserer Spezies „Mensch“. Vermutlich spielt der fitness-Gewinn durch Kooperationsfähigkeit eine entscheidende Rolle für die Evolution menschlicher Gehirnfähigkeiten überhaupt. Dieser Gewinn bezieht sich durchaus auch auf individuelle fitness, an der die genetische Selektion ansetzt: Es lohnt sich für den Einzelnen, sich als kooperativ darzustellen. „Good guys“ finden eher Kooperationspartner als „bad guys“, Menschen, die nach einem Streit versöhnungsbereit sind, fahren besser als dauerhaft unversöhnliche. Ein Mass an Anfangsvertrauen und Fairness auch gegenüber Unbekannten erhöht Kooperationschancen. Vermutlich gibt es eine pauschalierte, von bewussten Einzelabwägungen unabhängige Reziprozität in Form von Dispositionen zu ,low-cost-altruistic-actions“, man denke an die Höflichkeit oder die Gastlichkeit. Auf solche Weise beziehen neuere Linien soziobiologischen Denkens auch „freundliche“ Eigenschaften des Menschen ein.

Wesentlich für die Evolution des modernen Menschentyps vor vielleicht zweihunderttausend Jahren war, so die Vermutung, der Prozess der genetischen Weichenstellung, mit zunächst geringen Auswirkungen, die dann durch eine Vielzahl nachfolgender Mutationsschritte weiter ausgeprägt wurden - also ein phänotypischer Gradualismus und dennoch eine distinkte Rolle für spezifische Veränderungen im Genom. Weichenstellungen durch eine oder wenige Zufallsmutationen, die selten, aber nicht extrem unwahrscheinlich waren, konnten in der Folge zum Einzug neuer MetaEbenen der Informationsverarbeitung im Gehirn führen. Dabei ist besonders an die Neukombina- 
tion eines oder weniger Abschnitte der Genregulierung zu denken, die an der Ausbildung der Verschaltung der Neuronen in der Grosshirnrinde über weite Bereiche beteiligt sind. Die molekulare Genetik zeigt interessante Beispiele für „Rekrutierungen" von Abschnitten des Genoms durch Kombination in einen neuen funktionalen Kontext bei der Evolution der Organismen.

Diese Argumente für die Schlüsselrolle von eher seltenen und somit distinkten Genänderungen lassen sich durch Vergleiche mit Innovationen in der Geschichte der Technik stützen. Darin gibt es auch Beides, Kontinuität von Quantitäten ebenso wie qualitative Innovationen, zumal durch Neukombinationen. So zum Beispiel in der Schifffahrt: Die Segelschifffahrt entwickelte sich wesentlich durch die quantitative Vergrösserung der Segelfläche - grössere Segel, mehr Masten, mehr Segel pro Mast - die Dampfschifffahrt aber durch Neukombination von Elementen, die in anderem Kontext schon beträchtliche technische Reife erlangt hatten: Dampfmaschine und Schiffsrumpf; Eisen statt Holz für den Rumpf; die Schiffsschraube statt des Schaufelrades für den Antrieb. Gerade der Vergleich mit der Technikentwicklung legt es nahe, dass bei innovativen Vorgängen im Allgemeinen, zumal auch bei der Evolution menschlicher Gehirnfähigkeiten, Kontinuität und Singularität ihre Rolle spielten. Dabei ist mit distinkten Schritten der Mutation besonders dann zu rechnen, wenn es um die Neukombination von schon ausgereiften Teilfähigkeiten geht. Gerade diese Voraussetzung dürfte für die Evolution von Gehirnfähigkeiten des biologisch modernen Menschentyps auf der Basis von Teilfähigkeiten seiner Vorgänger in der Evolution zutreffen.

Es ist ganz generell wenig plausibel, Gehirnfähigkeiten, die bei der Menschwerdung entstanden, in erster Linie als Folge diffuser Selbstorganisation vergrösserter Gehirne anzusehen, wie das immer wieder behauptet wird. Nach der Erfindung des Dampfschiffes stiegen Schiffsgrössen um Faktoren um hundert, von Fultons ,Clermont“ bis zur berühmten „Titanic“; niemand aber würde den Dampfantrieb durch Selbstorganisation bei Zunahme von Schiffsgrössen erklären wollen. Primär war natürlich die neue Kombination von Dampfmaschine und Schiff. Dementsprechend dürften - auch, aber nicht nur bei der Menschwerdung - neue Kombinationen von Genabschnitten manche Entwicklungsmöglichkeiten eröffnet haben, durch die erst in der Folge Grosshirnvergrösserungen evolutionär lohnend werden konnten; ohne neue oder stark erweiterte Fähigkeiten hätte Vergrösserung angesichts des besonders hohen Energieverbrauchs von Nervensystemen nicht nur Vorteile in der Evolution gebracht.

Was die Funktionen des menschlichen Bewusstseins angeht, so habe ich auf eine - wenn nicht die - zentrale Rolle des bewussten planenden Denkens und Entscheidens hingewiesen. Diese beruht auf der Interpretation der gesamten von einer Person wahrgenommenen Situation, undzwar im Licht von Erfahrungen der Vergangenheit und möglichen Szenarien für die Zukunft einschliesslich ihrer emotionale Färbung und Bewertung; dafür wiederum sind die Representationen befürchteter bzw. erhoffter eigener Zustände von, im Wortsinne, entscheidender Bedeutung. Bei solchen mit Planung und Selbstbezug verbundenen Integrationsleistungen ist zwar der präfrontale Cortex ganz wesentlich beteiligt, sie umfassen aber insgesamt weite Bereiche des menschlichen Gehirns. Sie resultieren oft, aber nicht immer in bewusstem Erleben und machen wesentlich die Gesamtbefindlichkeit einer Person aus. Dabei sind zumeist nur die Ergebnisse der Integrationsleistungen, nicht aber alle vorgeschalteten Prozesse der Informationsverarbeitung und emotionalen Bewertung bewusst. Insbesondere werden wohl gerade solche Situationen ins Bewusstsein gehoben, in denen es Konflikte zwischen Motiven und wesentlich verschiedene Handlungsoptionen bei vergleichbarer emotionaler Gesamtbewertung gibt, sodass zwischen ihnen erst durch weitere Gehirnprozesse - nicht zuletzt auch mit Hilfe des bewusstes Denkens - zu entscheiden ist. Bewusstes Wissen, Denken und Fühlen ist so an der menschlichen Willensbildung essentiell beteiligt, zumindest in komplexen Situationen. Wegen ihrer Bedeutung für strategische Voraussicht ist die Zweckmässigkeit einer Evolution der Fähigkeiten des Bewusstseins aus biologischer Sicht nachvollziehbar. Ihre biologische Funktion an sich erklärt aber nicht in befriedigender Weise, wie Zustände und Vorgänge ins Bewusstsein gehoben werden und wie bewus- 
ste mit unbewussten Vorgängen zusammenwirken.

Die wissenschaftlichen Überlegungen und Forschungsergebnisse zum Thema „Bewusstsein“ sollten daher nicht den Blick auf die prinzipiellen Grenzen der Erkenntnis verstellen, die einer vollständigen naturwissenschaftlichen Erklärung des menschlichen Bewusstseins entgegenstehen. Auch Einsichten in seine evolutionären Ursprünge ergeben in sich noch kein umfassendes Verständnis von dessen stark verallgemeinerungsfähigen Potentialen. Das ist nicht viel anders als bei sehr allgemeinen technischen Erfindungen, etwa der des Rades: Die erste Erfindung von hölzernen Rädern für Wagen vor vielleicht 6000 Jahren impliziert keineswegs schon das ganze Spektrum der Entwicklungsmöglichkeiten des Konzepts „Rad“, etwa die Töpferscheibe, die Schiffsschraube, die Windmühle, die Gebetsmühle, Buddhas Rad der Lehre, das Fahrrad, das Zahnrad, das Feuerrad, das Glücksrad, das Kugellager und das Düsenaggregat...

Am wenigsten aber dürfen wir erwarten, dass uns die Neurobiologie sicher zu eindeutigen Antworten auf die tiefsten philosophischen Fragen führt, die mit menschlichem Denken und Bewusstsein verbunden sind. Das möchte ich an zwei Problemfeldern skizzieren, der Willensfreiheit und der Beziehung von Naturwissenschaft und Religion.

\section{Willensfreiheit, Hirnforschung und Grenzen objektiver Entscheidbarkeit: Was für nieman- den determiniert ist, ist nicht determiniert}

Wir empfinden uns in gewissem Maße als frei in der Wahl unserer Handlungen und verantwortlich für deren Folgen. Zwar läßt sich Wahlfreiheit mit soziologischen, psychologischen und philosophischen Argumenten anzweifeln, doch ändert dies nichts an dem Bewußtsein des einzelnen dafür, daß er Entscheidungsalternativen hat und wahrnimmt. Weitgehend unabhängig von theoretischen Auffassungen unterstellen wir lebenspraktisch eine Willensfreiheit des Menschen; ohne diese gäbe es kein wertbestimmtes soziales Verhalten. Gibt es Willensfreiheit wirklich und wenn ja, in welchem Sinne? Wieweit können wir überhaupt verantwortlich handeln? Wie vertragen sich das subjektive
Empfinden, zwischen verschiedenen Handlungsalternativen frei wählen zu können und der Anspruch der Gesellschaft, Rechenschaft für eine bestimmte Handlung zu verlangen, mit dem streng naturgesetzlichen Ablauf der Ereignisse im Menschen, einschließlich auch denjenigen in seinem Gehirn?

Diese Frage wird in jüngster Zeit besonders in Zusammenhang mit neuesten Ergebnissen neurobiologischer und neuropsychologischer Forschungen diskutiert. Sie weisen eine Vielfalt von Zusammenhängen von Aktivitäten in bestimmten Teilbereichen des menschlichen Gehirns mit höheren geistigen Fähigkeiten (einschliesslich ihrer emotionalen Korrelate) auf, so denen der Sprache, der Abstraktion, der strategischen Planung und der Empathie. Kein Zweifel, dass bewusstes Erleben mit Zuständen und Prozessen in unserem Gehirn auf das engste verbunden ist. Lassen die Naturgesetze dann überhaupt so etwas wie freien Willen $\mathrm{zu}$ ? Erhebliche Aufmerksamkeit fanden in jüngerer Zeit auch experimentelle Ergebnisse, nach denen die Vorbereitung willkürlicher Bewegungen, zum Beispiel die eines Fingers, bereits durch bestimmte Gehirnaktivitäten nachweisbar ist, ehe uns die Handlungsabsicht bewusst wird - Bruchteile einer Sekunde zuvor. Allerdings kann es danach noch ein bewusstes ,veto“ geben, sodass die Handlung unterbleibt. Zudem ist die Auslösung einfacher Bewegungen nicht prototypisch für die Vorbereitung bewusster Entscheidungen auf Grund planerischen Denkens, das für menschliche willentliche Entscheidungen eine so grosse Rolle spielt. Dennoch: Die Verschränkungen bewusster und unbewusster Vorgänge und ihre zeitlichen Beziehungen sind wohl komplexer, als man früher gedacht hatte. Lassen sich aber nun mit den Ergebnissen der Hirnforschung generelle Zweifel an der Willensfreiheit begründen?

Derartige Schlüsse wären mehr als voreilig. Da Bewusstsein, Wille und Freiheit keine Begriffe physikalisch begründeter Naturwissenschaft sind, folgt allein aus der Neurobiologie für die Willensfreiheit wohl gar nichts; durch Verbindungen der naturwissenschaftlichen Erkenntnisse mit Grundeinstellungen, Intuitionen und ,soft facts“aus anderen Bereichen ist es aber möglich, wenigstens $\mathrm{zu}$ begründeten Vermutungen zu gelangen. Diese 
allerdings hängen von der Wahl der zusätzlich hinzugezogenen Erkenntnisfelder ab.

Ich komme bei dieser Wahl auf die beiden schon erwähnten Grundeinstellungen zurück, die in diesem Zusammenhang nach meiner Ansicht besonderes Vertrauen verdienen: Konsequenten Physikalismus - keinesfalls kann unser Wille die Gültigkeit der physikalischen Gesetze in unserem Gehirn aushebeln - und entscheidungstheoretische Skepsis. Wesentlich ist, dass mit Erkenntnisgrenzen zu rechnen ist, zumal wenn Selbstbezug involviert ist: Wie ich schon erläutert habe, ist vermutlich eine vollständige Dekodierung physikalischer Gehirnzustände in Bezug auf psychische Zustände prinzipiell unmöglich. Nach dieser Auffassung sind unsere Willensentscheidungen zwar nichts unphysiologisches beziehungsweise antiphysikalisches, sie involvieren bewusste ebenso wie unbewusste Vorgänge (und warum soll es dabei nicht auch eine nachträgliche bewusste Entscheidung für eine vorgängig unbewusst vorbereitete Handlung geben können?). Aber unser Wille kann auch durch solche Innenfaktoren in uns mitbestimmt sein, die der Aussenanalyse durch Andere prinzipiell nicht vollständig zugänglich sind. Von solchen selbstbezogenen Innenfaktoren sind die erwähnten, mit dem Namen Libet verbundenen Experimente zur Fingerbewegung sehr weit entfernt. Welche philosophischen Auffassungen auch immer wir hierzu vertreten - stringente Schlüsse der Neurobiologie allein in Bezug auf das Willensfreiheitsproblem sind auch auf der Basis der LibetExperimente nicht möglich.

Allerdings meine ich, dass Grenzen der Entscheidbarkeit mit rigoros deterministischen Auffassungen unverträglich sind: Was für niemanden determiniert ist, ist nicht determiniert. Unser Gehirn unterliegt zwar den gleichen physikalischen Gesetzen wie eine Maschine; aber eine Maschine, die wir vollkommen verstehen, leistet nicht alles wie unser Gehirn, und eine Maschine, die alles leistet wie unser Gehirn, würden wir ebenso wenig vollständig verstehen wie das Gehirn selbst. Deshalb können Selbstaussagen über bewusste Zustände und Vorgänge im Prinzip über das hinausführen, was durch noch so raffinierte objektive Methoden durch Aussenstehende herauszubringen wäre.
Allgemein dürften Einsichten über Grenzen der Dekodierung der Gehirn-Geist-Beziehung durchaus mehr Beachtung durch Historiker, Philosophen und Sozialwissenschaftler, Journalisten und Politiker, Ankläger und Richter verdienen - undzwar in Richtung auf Zurückhaltung im Urteil: Einem verlässlichen Einstieg in fremdes Bewusstsein, fremde Gedanken, fremdes Wissen und fremde Motive sind vermutlich unüberwindliche, epistemologisch robuste Grenzen gesetzt. Perfektes „mind-reading“ gibt es schlechthin nicht - zum Glück.

Was die Willensfreiheit angeht, so bleiben Deutungsspielräume, und die bevorzugten Deutungen sind auch eine Frage der Lebenskunst. Wollen wir wirklich die erlebte Willensfreiheit als schlechthin betrügerische Illusion ansehen? In einer nicht ganz streng und ernst gemeinten Erklärung hat hierzu der Verhaltensforscher Konrad Lorenz einmal bemerkt, das mit dem Determinismus sei doch eigentlich nicht so schwierig. Es gibt Optimisten und Pessimisten; Pessimisten empfinden meist fatalistisch und neigen zum Determinismus, Optimisten setzen eher auf Selbstvertrauen und dabei auf Freiheit ihres Willens.

\section{Naturwissenschaft, Religion und die philosophische Mehrdeutigkeit der Welt}

Insgesamt ergibt die Neurobiologie durchaus wesentliche Erkenntnisse über unsere Spezies „Mensch“, die auch von den Geisteswissenschaften zu deren Vorteil zu integrieren sind. Die neuere Biologie widerlegt viele Vorstellungen zum Beispiel über ausserphysikalische biologische Prinzipien, sie erhellt Grund- und Randbedingungen unseres Verständnisses vom Menschen. Die metatheoretische Mehrdeutigkeit unseres Wissens auf der philosophischen und kulturellen Ebene wird aber dadurch nicht aufgehoben. Nicht jede, aber mehr als eine Deutung ist mit naturwissenschaftlichem Wissen logisch verträglich. Das gilt wohl auch für das Verhältnis von Naturwissenschaft und Religion.

Noch vor wenigen Generationen sahen die meisten Intellektuellen ein Absterben der als vorwissenschaftlich angesehenen Religionen zugunsten eines wissenschaftlich dominierten Welt- 
verständnisses voraus. Das prognostiziert heute kaum noch jemand. Die dramatische Erweiterung unseres Wissens im 20. Jahrhundert war engstens mit Selbstbegrenzung naturwissenschaftlichen Denkens durch die wissenschaftliche Reflexion der eigenen Voraussetzungen verbunden, und dies wiederum führt zu einer offeneren Sicht auf religiöse Weltdeutungen. Dies liegt nicht nur, aber auch daran, dass liberale, undogmatische Versionen theologischen Denkens mit Wissenschaft und logischem Denken vereinbar sind, wenn die Selbstbegrenzung der Letzteren beachtet wird. So ist die religiöse Deutung der Ordnung der Natur als Schöpfung Gottes und des Menschen als Ebenbild des Schöpfers im geistigen, kreativen Sinn nicht nur logisch konsistent mit wissenschaftlichem Denken; sie bildet auch eine, wenngleich ,weiche“, Erklärung dafür, warum die gesetzmässige Ordnung doch so unerwartet weit dem menschlichen Denken zugänglich ist, wie es die Geschichte des Kulturprodukts „Naturwissenschaft" aufzeigt. Wie kommt der menschliche Geist zu der Fähigkeit, die Formel $E=m c^{2}$ zu konzipieren und zu bestätigen? Evolutionsbiologische Erklärungen, die bei den Bedingungen von Jägerund Sammlerkulturen der Steinzeit ansetzen, tun sich mit der Beantwortung dieser Frage nicht eben leicht. Das Unverständlichste am Universum ist im Grunde, so Einstein, dass wir es verstehen könnenjedenfalls weitergehend verstehen, als man früher gemeint hat. Vereinbarung von wissenschaftlichem mit religiösem Denken bleibt aber eine Option und kein Muss. Es kann nicht verwundern, dass es unter Wissenschaftlern viele gibt, die Religion aus berechtigtem Ärger über religiösen Fundamentalismus nach Art des Kreationismus ablehnen. Das ist verständlich, aber auch in ablehnenden Argumenten finden sich nicht selten ihrerseits fundamentalistische, unduldsame und dogmatische Züge. Die Aufklärung des 18. und des Anfangs des 19. Jahrhunderts hat begründet, dass religiöse Auffassungen nicht naturwissenschaftliche Erkenntnisse widerlegen können. Die nachfolgende Aufklärung über die Aufklärung, mit Ausläufern bis in die Gegenwart, impliziert dann aber auch, dass die Naturwissenschaft nicht die Religionen widerlegt, jedenfalls nicht in deren ihrerseits aufgeklärten Formen. Die Einstellung zu
Religion ist nicht allein auf Grund wissenschaftlicher Kriterien zu entscheiden, sie hängt von individuellen, sozialen und kulturellen Voraussetzungen ab und ist nicht zuletzt eine Frage der Weisheit und der Lebenskunst. Die realistische Prognose lautet, dass agnostische und religöse Weltdeutungen auf Dauer koexistieren werden. Was das gesellschaftliche Wohlergehen betrifft, so hängt sehr viel davon ab, dass jeweils innerhalb religiöser wie auch agnostischer Strömungen die liberalen und toleranten gegenüber den fundamentalistischen und dogmatischen die Oberhand gewinnen und behalten.

Was nun die Rolle der Neurobiologie in diesem Kontext angeht, so kann sie zweifellos allgemeine Beiträge zum Selbstverständnis des Menschen leisten, indem sie Gehirneigenschaften $\mathrm{zu}$ erklären hilft. Eindeutige Antworten auf die damit verbundenen grundsätzlichen, philosophischen Fragen sind aber nicht zu erwarten, und das gilt besonders für das Verständnis von Religion. Was können wir lernen, wenn wir neurobiologische Prozesse im Kontext religiöser Gedanken, Gefühle und Erlebnisse beobachten, etwa durch aktivitätsabhängiges Neuro-Imaging? Manches ist ja schon vor solchen Untersuchungen klar: Dass all dies mit Gehirnaktivitäten verbunden ist; und dass, soweit es sich um Fähigkeiten handelt, die zum Beispiel Schimpansen nicht haben, dem dann auch Unterschiede in Gehirnvorgängen, vermutlich auch von Gehirnstrukturen zwischen Mensch und Schimpanse zugrunde liegen, und nicht zuletzt auch Unterschiede von Genen, die die Gehirnentwicklung steuern und in der menschlichen Evolution gebildet oder verändert wurden - so schwer dies auch im Einzelnen nachweisbar sein mag. Sodann zeigen soziobiologische Überlegungen, dass die religiösen Fähigkeiten Leben und Überleben befördert haben, und damit wird ihre ursprüngliche Entwicklung evolutionsbiologisch einsichtig. Und doch wäre es ein grosser Irrtum zu glauben, man könne auf diesem Wege Religion vollständig erklären oder gar wegerklären, sozusagen entlarven. Bei naturwissenschaftlichen Aussagen, zum Beispiel zur Evolution des Lebens auf der Erde, hat die Biologie gegenüber tradierten Überlieferungen der Religionen das letzte Wort; über die Wahrheitsansprüche philosophischer, zumal me- 
taphysischer Voraussetzungen menschlichen Denkens aber kann die Biologie nicht verbindlich entscheiden. Wesentlich für unser Selbstverständnis bleibt, dass allgemeinen Fähigkeiten der Spezies Mensch weit über den evolutionären Anlass ihrer Entstehung hinausführen können, und dieser Überschuss fällt in die Domäne von Kulturgeschichte und Philosophie, nicht von Biologie.

Man kann sich dies an einer der philosophisch tiefsten und allgemeinsten Fragen klarmachen, die unsere Intuitionen zugunsten oder zu Ungunsten eines religiösen Weltverständnisses wesentlich mitbestimmt: Gibt es eine Ordnung der Welt, des Denkens und der Dinge auch ohne uns Menschen, eine Ordnung, die wir sie mit unserem evolvierten Gehirn im Laufe der letzten Jahrtausende der Kulturgeschichte dann entdeckt und mehr oder weniger gut verstanden haben? Inwieweit und inwiefern ist diese Ordnung vom menschlichen Erkenntnisapparat strukturiert? Ist sie vielleicht überhaupt erst eine Konstruktion menschlicher Gehirne, also eine Ordnung, die es ohne uns Menschen gar nicht geben würde? Mit den Mitteln der Neurobiologie sind diese Fragen nicht entscheidbar: Es gäbe ja in jedem Fall menschenspezifische, wohl auch neurobiologisch nachweisbare Gehirnaktivitäten, die mit religiösen Gedanken und Empfindungen verbunden sind, ganz unabhängig davon, ob die Weltordnung nun von Gott in wesentlichen Zügen am Anfang der Dinge geschaffen wurde oder ob sie ausschliesslich erst von uns Menschen konstruiert ist.

Zum Vergleich lässt sich das gleiche Argument auf einen weniger emotionalen Kontext übertragen, auf ein wenn nicht das philosophisch hintergründigste Grundproblem der Mathematik: Gibt es mathematische Wahrheiten, mentale Wirklichkeiten, gibt es zum Beispiel, wie es die idealistische Philosophie behauptet, die platonischen Körper auch unabhängig von uns Menschen? Oder sind sie nur Konstruktionen unseres menschlichen Denkens? Machen wir hierzu ein Gedankenexperiment: Schieben wir einen klugen Menschen in einen Computertomographen, der die Gehirnaktivitäten misst und lokalisiert, und fragen ihn nach den Primzahlen zwischen 10 und 20. Er sagt 11, 13, 17, 19; die nächste ist 23. Dann fragen wir, $\mathrm{ob}$ es endlich oder unendlich viele Primzahlen gibt und erzählen ihm den Beweis dafür, dass es unendlich viele gibt: Gäbe es nur endlich viele, so könnten wir sie alle miteinander multiplizieren und hätten wieder eine Zahl. Zählen wir eins da$\mathrm{zu}$, haben wir aber noch eine Primzahl - die als vollständig vorausgesetzte Liste der Primzahlen war gar nicht vollständig! Also kann es nicht nur endlich viele Primzahlen geben. Kennt nun unser Proband diesen Beweis noch nicht oder nicht mehr, wird er ihn erhellend und witzig finden. Alles nur Konstruktion? Dann fragen wir als nächstes, ob es endlich oder unendlich viele Primzahlpärchen wie 11,13 oder 17,19 gibt - eines der schwierigsten Probleme der Zahlentheorie. Wenn tatsächlich alles nur Konstruktion ist, dann konstruieren wir doch einfach das Ergebnis! Gerade das geht aber nicht, es handelt sich, soviel ich weiss, um ein immer noch ungelöstes Problem. Schliesslich befreien wir unseren Kollegen aus dem Tomographen und werten die Diagramme über seine Gehirnprozesse aus. Wir lernen einiges über den Umgang des menschlichen Gehirns mit Zahlen, aber sicher nichts darüber, ob wir sie nur konstruieren, oder ob es Wahrheiten über sie auch ohne uns gibt - an Hirnströmen allein würde sich die philosophische Wahrheit nicht zeigen.

Und hier liegt die Parallelität mit der genannten Grundfrage zur Religion: Gibt es eine Ordnung der Welt, der Dinge und des Geistes nur durch und in uns oder auch ohne und vor uns - auch das werden wir im Prinzip nicht durch neurobiologische Forschung entscheiden können. Wir bleiben in solchen Fragen nicht zuletzt auf Lebenskunst und philosophische Vernunft angewiesen, und beide sprechen nach meiner Ansicht gegen einseitig konstruktivistische Auffassungen. Auch ein besseres Verständnis der evolutionären und kulturgeschichtlichen Ursprünge der Religionen, so interessant entsprechende Erkenntnisse auch sind, kann die Potentiale religiöser Beiträge zum menschlichen Selbst- und Weltverständnis nicht erschöpfen - ebenso wenig wie die schon erwähnte Erfindung des hölzernen Scheibenrades für Transportkarren vor vielleicht 6000 Jahren das ganze, vielfältige Potential des mentalen Konzeptes „Rad“ erfasst, das sich erst in einer langen kulturgeschichtlichen Entwicklung zeigte. 


\section{Abschließende Betrachtungen}

Die großen Fortschritte der Neurobiologie in der Gegenwart machen es psychologisch verständlich, daß heutzutage viele daran Beteiligte an die asymptotische Lösbarkeit aller wichtigen Fragestellungen glauben. So haben es die Physiker und Mathematiker in ihren Gebieten um 1900 getan. Dann aber, um 1927, war diese Auffassung durch die Entwicklung der Quantenphysik widerlegt worden. Bestimmte atomare Ereignisse können im Prinzip nicht genau vorausberechnet werden, gleichgültig, wieviel Mess- und Berechnungsaufwand wir treiben, und diese Beschränkung ist selbst ein Naturgesetz! Und noch 1930 erklärte der grosse Mathematiker Hilbert, es gäbe keine unlösbaren mathematischen Probleme. Ein Jahr später, mit Gödels Unentscheidbarkeitstheoremen, war es auch damit vorbei.

In der Gegenwart versprechen sich zudem manche Physiker Lösungen des Bewusstseinsproblems von einer künftigen, erweiterten Physik. Bei aller Skepsis: Ausgeschlossen ist das nicht, aber es ist wohl auch nicht sehr wahrscheinlich. Und dann gibt es viele recht spekulative Überlegungen, wieweit die neuen Einsichten in die Quantenwelt Folgerungen, wenn nicht gar Lösungen auch zum Bewusstseinsproblem bieten könnten. Ich habe diese Ansätze nicht ernsthaft verfolgt und kann dazu deshalb auch kein fundiertes Urteil abgeben. Das Gehirn ist kein Quantencomputer; ob es Funktionen gibt, bei denen Quanteneffekte wie Verschränkung, Dekoherenz oder Nichtlokalität eine unmittelbare Rolle spielen, ist offen und erscheint derzeit nicht sonderlich plausibel.

Hinter solchen merkwürdigen quantenphysikalischen Phänomenen könnten sich aber allgemeinere Merkmale physikalisch-mathematischen Naturverständnisses verbergen. Dies betrifft nicht zuletzt den Schlüsselbegriff „Information“, der in Diskussionen um die Grundlagen der modernen Physik eine wesentliche Rolle spielt. Dass die übergeordneten, oft so hintergründigen und dabei anti-intuitiven erkenntnistheoretischen Merkmale der Quantenphysik hinsichtlich der Beziehung von Einsicht und Realität - und damit der Beziehung des Mentalen zum Materiellen - auch für das Gehirn-Geist-Problem relevant, zumindest aber lehrreich sein könnten, dafür sollten wir aufgeschlossen sein. Es ist mehr als zweifelhaft, die Gehirnphysik als Argument gegen die Willensfreiheit in Anspruch zu nehmen, indem man mehr oder weniger implizit auf Intuitionen der mittlerweile etwas angestaubten deterministischen Mechanik des 19. Jahrhunderts zurückgreift.

Stattdessen dürften entscheidungstheoretische Aspekte der Gehirn - Geist - Beziehung unsere besondere Beachtung verdienen: Verstehen wir Grenzen der Berechenbarkeit auf Grund der Endlichkeit physikalisch möglicher Informationsverarbeitung in einem endlichen Universum als erkenntnistheoretisch robuste Grenzen des möglichen Wissens, so erscheinen psychische Zustände zwar als Funktionen physikalischer Gehirnzustände, aber es handelt sich vermutlich nicht um vollständig berechenbare Funktionen. Informationstheoretisch gibt es vielmehr einen Grad von prinzipiell unüberwindlicher Unbestimmtheit. Darin liegt auch ein Grad Freiheit in dem Sinne, dass eigene psychische Vorgänge von Anderen nicht unbegrenzt durchschaubar und steuerbar sind: Willensfreiheit besteht - in Grenzen - in einer Autonomie interner Vorgänge in einer handelnden Person.

Ganz allgemein vermute ich, dass es letztlich in Bezug auf die Erklärung von Bewußtsein prinzipielle Grenzen der Erkenntnis gibt, ähnlich wie in der Physik und Mathematik. Dabei geht es auch um die Problematik von Selbstbezug, um Grenzen des Bewußtseins von Bewußtsein. Ich sehe allerdings, ähnlich wie schon Cusanus im 15. Jahrhundert, Erkenntnisse über Grenzen der Erkenntnis eher positiv: Sie sind Folgen der Erweiterung, nicht der Verengung unseres Denkens. Menschliches Bewusstsein entstand zwar als Ergebnis biologischer Evolution, aber es begründet sehr allgemeine Fähigkeiten, die über den Anlass der Entstehung zu Jäger- und Sammlerzeiten weit hinausführen und sich erst in der kulturellen Entwicklung voll entfalteten, wie z.B. das strategische Denken und die kognitionsgestützten Empathie. Ich habe Gründe für die Vermutung erörtert, dass bei der Evolution des biologisch modernen Menschentyps vor vielleicht 200000 Jahren neue Meta - Ebenen der Informationsverarbeitung - ein Stichwort wäre hier Selbstbezug - in die Verschaltungen des neuronalen Netzes eingezogen wurden. 
Naturphilosophisch gesehen führt dies $\mathrm{zu}$ analogen Problemen mit ähnlichen entscheidungstheoretischen Konsequenzen, wie wir sie beim Übergang von armen zu reichen logischen Systemen kennen, besonders bei der Erweiterung der leistungsschwachen Aussagenlogik zur leistungsstarken Prädikaten- bzw. Quantorenlogik: Im Gegensatz zum schwachen lässt sich das starke System nicht mehr aus sich selbst heraus absichern, hat aber dafür im positiven Sinne ein sehr weites, offenes Potential. $\mathrm{Zu}$ den erstaunlichsten Potentialen des menschlichen Denkens gehört die moderne Naturwissenschaft, dieses späte Produkt europäischer Kulturgeschichte. Sie lässt sich wirklich nicht mehr vernünftig auf Evolutionsvorteile zu Jäger- und Sammlerzeiten zurückführen, und doch verbindet sie in erstaunlicher und überraschender Weise das menschliche Denken mit der Ordnung der Vorgänge und der Dinge: Eine spannende Herausforderung im Grenzbereich von Natur- und Geisteswissenschaften, was das Verständnis - und Selbstverständnis - menschlichen Bewusstseins angeht.

\section{Bibliographie}

Eine systematische Literaturliste zu den zahlreichen, hier angesprochenen Themen würde über den Rahmen dieses Essays hinausgehen. Um die Beziehung von Wissenschaft und Menschenbild, nicht zuletzt um die knappe Ressource Gemeinsinn, geht es in meinem Buch Gierer 1998; dort finden sich, nach Teilthemen geordnet, Literaturhinweise auf den Seiten 289-312. Zu Grenzen der Dekodierbarkeit der Gehirn-Geist-Beziehung möchte ich verweisen auf den Artikel Gierer 2008. Technikgeschichtliche Argumente zur Gehirnevolution sind Themen in Gierer 2004.

Gierer, Alfred, 1998 Im Spiegel der Natur erkennen wir uns selbst - Wissenschaft und Menschenbild, Reinbek: Rowohlt.

Neuformatiert online unter http://www.eb.tuebingen.mpg.de/departments/ former-departments/a-gierer/Im\%20Spiegel.pdf (Literaturhinweise S. 177-191).

Volltext auch unter http://edoc.bbaw.de/.
Gierer, Alfred, 2004, "Human brain evolution, theories of innovation, and lessons from the history of technology", in: Journal of Biosciences 29, Nr. 3, S. 235-244. http://www.eb.tuebingen.mpg.de/departments/ former-departments/a-gierer/BrainEvolution.pdf

Gierer, Alfred, 2008, "Brain, mind and limitations of a scientific theory of human consciousness", in: BioEssays 30, S. 499-505. http://www.eb.tuebingen.mpg.de/departments/ former-departments/a-gierer/brain-mindbioess.pdf 